Pacific Journal of Mathematics

HERMITE CHARACTER SUMS 


\title{
HERMITE CHARACTER SUMS
}

\author{
RONALD J. EvANS
}

\begin{abstract}
Analogues over finite fields are presented for the major formulas in the theory of classical Hermite functions.
\end{abstract}

1. Introduction. Character sum analogues over finite fields of the most important transformation and summation formulas for ${ }_{2} F_{1}$ and ${ }_{3} F_{2}$ hypergeometric series have recently been formulated by Greene [11], [11A]. The power of this theory is demonstrated, for example, by the evaluation it yields [12] of the double sum of Legendre symbols

$$
\sum_{x, y(\bmod p)}\left(\frac{x y(x+1)(y+1)(x+y)}{p}\right) \text {. }
$$

This evaluation proves a conjecture in $[9$, p. 370] and solves the problem of finding explicitly the number of rational points $(\bmod p)$ on the surface $z^{2}=\left(x^{2}+1\right)\left(y^{2}+1\right)\left(x^{2}+y^{2}\right)$, a problem some algebraic geometers had worked on without success.

Character sum analogues of the important formulas for orthogonal polynomials are potentially as useful as those for hypergeometric series, so a systematic study should be made. Indeed, many character sums studied in the literature are analogues of special functions, e.g., the generalized Kloosterman sum (see (2.5), Theorem 2.6, and, say, [10], [21A, p. 253]).

In this paper, the focus is on analogues of Hermite polynomials, namely Hermite character sums $H_{N}(x)$ defined in (2.1). Each of the theorems in $\S 4$ is an analogue over finite fields of a classical formula stated just above it. The classical formulas are stated without conditions of validity; such conditions are often unrelated to the unpredictable conditions of validity for the finite field formulas.

It is not always possible to give proofs of the finite field formulas which parallel classical proofs. This is because no satisfactory analogues of limits, first derivatives, logarithms, and three term recurrence relations are known. It would be of great importance to find a unified approach which simultaneously explains formulas for orthogonal polynomials and the analogues over finite fields. Perhaps this will be accomplished by connecting the polynomials with Lie groups having counterparts over finite fields. 
Theorems 4.24 and 4.36 are particularly elegant and interesting. Surprisingly, the former holds with absolutely no restrictions on the characters $A, B$, and $C$.

In $\S 3$, multivariable Hermite sums are defined and a biorthogonality relation is proved. In $\$ 5$, an analogue of the associated Hermite polynomial [1] is briefly discussed, and an example is given to show how finite field analysis may be used to construct explicit formulas for classical special functions. The mysterious fact that such a technique generally works reflects the beauty and unity of mathematics.

For some recent work related to finite field analogues of classical formulas for special functions, see references [7]-[9], [11]-[16], [19], and [21]. This subject of course dates back a long time. As early as 1837, Jacobi [18, p. 257] had been aware of a finite field analogue of the Gauss multiplication formula for the gamma function [25, p. 26]. Jacobi did not have available the tools needed to prove his formula, and over a century went by before a proof was provided by Davenport and Hasse [5]. No elementary proof is known, but see [3, §8], [13].

2. Definitions, notation, and preliminary results. Let $q$ be a positive integral power of an odd prime $p$. The finite field of $q$ elements is denoted by $G F(q)$. The capital letters $A, B, C, M, N$ are reserved for multiplicative characters on $G F(q)$, but 1 and $\phi$ will denote the trivial and quadratic characters, respectively. Write $\Sigma_{x}$ to denote the sum over all $x \in G F(q)$, and write $\Sigma_{N}$ to denote the sum over all $q-1$ characters $N$ on $G F(q)$. For $x \in G F(q), \operatorname{Tr}(x)$ denotes the trace of $x$ from $G F(q)$ to $G F(p)$, and $\zeta^{x}$ denotes $\exp (2 \pi i \operatorname{Tr}(x) / p)$. If $x \in G F(q)$, let $\bar{x}$ be the multiplicative inverse of $x$ when $x \neq 0$ and let $\bar{x}=0$ when $x=0$. The expression $\zeta^{x / 2}$ means $\zeta^{x \overline{2}}$, not $\exp (2 \pi i \operatorname{Tr}(x) / 2 p)$. Define $\bar{N}$ by $N \bar{N}=1$.

Analogous to the gamma function

$$
\Gamma(n)=\int_{0}^{\infty} x^{n} e^{-x} \frac{d x}{x}
$$

is the Gauss sum

$$
G(N)=\sum_{x} N(x) \zeta^{x}
$$

and analogous to the beta function

$$
\beta(m, n)=\int_{0}^{1} x^{m}(1-x)^{n} \frac{d x}{x(1-x)}
$$

is the Jacobi sum

$$
J(M, N)=\sum_{x} M(x) N(1-x) .
$$


For some basic properties of these sums, see [17, Ch. 8]; e.g., for $A \neq 1$, $M N \neq 1, G(A) G(\bar{A})=q A(-1), J(M, N)=G(M) G(N) / G(M N)$.

We wish to define character sum analogues of Hermite functions $H_{n}(x)$, Laguerre functions $L_{n}^{a}(x)$, Legendre functions $P_{n}(x)$, and Bessel functions $J_{n}(x), K_{n}(x)$, motivated by the familiar integral representations

$$
\begin{array}{ll}
H_{n}(x)=\frac{1}{\Gamma(-n)} \int_{0}^{\infty} e^{-u^{2}-2 u x} u^{-n} \frac{d u}{u} & {[\mathbf{2 0},(10.5 .2)]} \\
L_{n}^{a}(x)=\frac{1}{2 \pi i} \int_{C}(u+1)^{n+a} u^{-n} e^{-u x} \frac{d u}{u} & {[\mathbf{2 0}, \mathrm{p} .77]} \\
P_{n}(x)=\frac{1}{2 \pi i} \int_{C}\left(1-2 x u+u^{2}\right)^{-1 / 2} u^{-n} \frac{d u}{u} & {[\mathbf{2 0}, \mathrm{p} .45]} \\
J_{n}(x)=\frac{1}{2 \pi i} \int_{C} u^{-n} e^{x\left(u-u^{-1}\right) / 2} \frac{d u}{u} & {[\mathbf{2 0},(5.10 .7)]} \\
K_{n}(x)=\frac{1}{2} \int_{0}^{\infty} u^{-n} e^{-x\left(u+u^{-1}\right) / 2} \frac{d u}{u} & {[\mathbf{2 0},(5.10 .25)]}
\end{array}
$$

Thus for $x \in G F(q)$, define the Hermite character sum

$$
H_{N}(x)=\frac{1}{G(\bar{N})} \sum_{u} \bar{N}(u) \zeta^{u^{2}+2 u x},
$$

the Laguerre character sum

$$
L_{N}^{A}(x)=q^{-1} \sum_{u} \bar{N}(u) A N(1+u) \zeta^{x u}
$$

the Legendre character sum

$$
P_{N}(x)=q^{-1} \sum_{u} N(u) \phi\left(1-2 x u+u^{2}\right),
$$

and the Bessel character sums

$$
\begin{gathered}
J_{N}(x)=q^{-1} \sum_{u} N(u) \zeta^{x(u-\bar{u}) / 2}, \\
K_{N}(x)=\sum_{u} N(u) \zeta^{x(u+\bar{u}) / 2} .
\end{gathered}
$$

(Note that $K_{N}(x)$ is a generalized Kloosterman sum.) Confluent hypergeometric character sums $\Psi(A, B ; x)$ and $\Phi(A, B ; x)$ (cf. [20, (9.11.6), (9.11.1)]) can be defined as multiples of $L_{\bar{A}}^{B}(x)$, as follows:

$$
\begin{gathered}
\Psi(A, B ; x)=\frac{q}{G(A)} L_{\bar{A}}^{B}(x), \\
\Phi(A, B ; x)=\frac{q A(-1)}{J(A, B \bar{A})} L_{\bar{A}}^{B}(x) .
\end{gathered}
$$


Define an operator $D^{N}=D_{x}^{N}$ on the set of complex functions $F$ on $G F(q)$ by

$$
D^{N} F(x)=\frac{1}{G(\bar{N})} \sum_{t} \bar{N}(t) F(x-t)
$$

Thus $D_{x}^{N}$ is the analogue of the $n$th derivative with respect to $x$ (cf. Cauchy's integral formula for $f^{(n)}(x)$ ). We next prove four theorems involving $D^{N}$. The first gives an analogue of composition of derivative operators.

THEOREM 2.1. For a function $F: G F(q) \rightarrow \mathbf{C}$,

$$
D^{1} F(x)=F(x)-\sum_{t} F(t),
$$

$$
D^{N} D^{\bar{N}} F(x)=F(x)-q^{-1} \sum_{t} F(t) \quad \text { for } N \neq 1,
$$

and

$$
D^{N} D^{M}=D^{N M} \quad \text { for } N M \neq 1
$$

Proof. By (2.8), one easily proves (2.9). Now,

$$
\begin{aligned}
L & :=G(\bar{N}) G(\bar{M}) D^{N} D^{M} F(x)=\sum_{s, t} \bar{M}(s) \bar{N}(t) F(x-t-s) \\
& =\sum_{s, t} \bar{M}(s) \bar{N}(t-s) F(x-t) \\
& =\sum_{t \neq 0} \sum_{s} \bar{M}(s) \bar{N}(t-s) F(x-t)+N(-1) F(x) \sum_{s} \overline{M N}(s) \\
& =J(\bar{M}, \bar{N}) \sum_{t} \overline{M N}(t) F(x-t)+N(-1) F(x) \sum_{s} \overline{M N}(s) .
\end{aligned}
$$

If $M=\bar{N} \neq 1$, then $J(\bar{M}, \bar{N})=-N(-1)$ and $G(\bar{N}) G(\bar{M})=N(-1) q$, so

$$
\begin{aligned}
L & =N(-1)\left\{-\sum_{t \neq 0} F(x-t)+(q-1) F(x)\right\} \\
& =N(-1)\left\{q F(x)-\sum_{t} F(t)\right\}
\end{aligned}
$$

and (2.10) follows. If $M \neq \bar{N}$, then $J(\bar{M}, \bar{N})=G(\bar{M}) G(\bar{N}) / G(\bar{M} \bar{N})$, so by (2.8), $L=G(\bar{M}) G(\bar{N}) D^{N M} F(x)$ and (2.11) follows.

The next theorem gives an analogue of Leibniz's rule. 
Theorem 2.2. If $E: G F(q) \rightarrow \mathbf{C}$ and $F: G F(q) \rightarrow \mathbf{C}$, then

$$
D^{N}(E(x) F(x))=\sum_{M} \frac{G(\bar{M}) G(M \bar{N})}{(q-1) G(\bar{N})}\left(D^{M} E(x)\right)\left(D^{N \bar{M}} F(x)\right) \text {. }
$$

Proof. By (2.8), the right side above equals

$$
\begin{aligned}
& \sum_{M} \frac{1}{(q-1) G(\bar{N})} \sum_{s, t \neq 0} \bar{M}(s) M \bar{N}(t) E(x-s) F(x-t) \\
& \quad=\frac{1}{G(\bar{N})} \sum_{s, t \neq 0} \bar{N}(t) E(x-s) F(x-t) \cdot \frac{1}{(q-1)} \sum_{M} M(t / s) \\
& \quad=\frac{1}{G(\bar{N})} \sum_{t} \bar{N}(t) E(x-t) F(x-t)=D^{N}(E(x) F(x)) .
\end{aligned}
$$

The next theorem gives an analogue of $n$-fold integration by parts.

Theorem 2.3. Let $E: G F(q) \rightarrow \mathrm{C}$ and $F: G F(q) \rightarrow \mathbf{C}$. Then

$$
\sum_{x} E(x) D^{N} F(x)=N(-1) \sum_{x} F(x) D^{N} E(x) .
$$

Proof. By (2.8),

$$
\begin{aligned}
G(\bar{N}) & \sum_{x} E(x) D^{N} F(x)=\sum_{u} E(u) \sum_{t} \bar{N}(t) F(u-t) \\
= & \sum_{x} F(x) \sum_{u} E(u) \bar{N}(u-x) \\
= & \sum_{x} F(x) \sum_{t} E(x-t) \bar{N}(-t)=G(\bar{N}) N(-1) \sum_{x} F(x) D^{N} E(x) .
\end{aligned}
$$

The next theorem is the analogue of the Taylor expansion.

TheOREM 2.4. Let $F: G F(q) \rightarrow \mathrm{C}$ and fix $a \in G F(q)$. For $x \neq a$,

$$
F(x)=\left.\sum_{N} \frac{G(\bar{N})}{q-1} D^{N} F(x)\right|_{x=a} N(a-x) .
$$

Moreover, this expansion is unique in the sense that if

$$
0=\sum_{N} R(N) N(a-x)
$$

for all $x$, then $R(N)=0$ for all $N$. 
Proof. For $x \neq a$,

$$
\begin{gathered}
\left.\sum_{N} \frac{G(\bar{N})}{q-1} D^{N} F(x)\right|_{x=a} N(a-x)=\sum_{N} \frac{N(a-x)}{q-1} \sum_{t} \bar{N}(t) F(a-t) \\
=\sum_{t \neq 0} F(a-t) \cdot \frac{1}{q-1} \sum_{N} N\left(\frac{a-x}{t}\right)=F(x) .
\end{gathered}
$$

To prove the statement on uniqueness, multiply both sides of the equality $0=\sum_{N} R(N) N(a-x)$ by $\bar{M}(a-x)$ and then sum over $x$.

The next theorem gives an analogue of Fourier inversion. We omit the easy proof.

TheOREM 2.5. Let $F: G F(q) \rightarrow$ C. Then $F(x)=\Sigma_{a} Q(a) \zeta^{a x}$, where $Q(a)=q^{-1} \sum_{u} F(u) \zeta^{-a u}$. Moreover, this expansion is unique in the sense that if $0=\sum_{a} R(a) \zeta^{a x}$ for all $x$, then $R(a)=0$ for all $a$.

The next theorem is the analogue of

$$
K_{1 / 2}(x)=\sqrt{\frac{\pi}{2 x}} e^{-x} \quad[20,(5.8 .5)] .
$$

It evaluates Saliẻ's sum over $G F(q)$; see Mordell [23], [24].

THEOREM 2.6. For all $x$,

$$
K_{\phi}(x)=\phi(2 x) G(\phi)\left(\zeta^{x}+\zeta^{-x}\right)
$$

Proof. By the uniqueness assertion in Theorem 2.5, it suffices to show that for all $a$,

$$
\begin{aligned}
Q(a) & :=q^{-1} \sum_{x} K_{\phi}(x) \zeta^{-a x} \\
& =q^{-1} \phi(2) G(\phi) \sum_{x} \phi(x)\left(\zeta^{x(1-a)}+\zeta^{x(-1-a)}\right) .
\end{aligned}
$$

The left side of (2.12) equals

$$
q^{-1} \sum_{t} \phi(t) \sum_{x} \zeta^{x(t+\bar{t}-2 a) / 2}=\sum_{\substack{t \\ t+\bar{t}=2 a}} \phi(t),
$$

and the right side of (2.12) equals

$$
\begin{gathered}
q^{-1} \phi(2) G(\phi)\{G(\phi) \phi(1-a)+G(\phi) \phi(-1-a)\} \\
=\phi(2 a+2)+\phi(2 a-2) .
\end{gathered}
$$


If $\phi\left(a^{2}-1\right)=-1$, then the expressions in (2.13) and (2.14) vanish; if $\phi\left(a^{2}-1\right)=0$, these expressions equal $\phi(a)$. Finally, assume that $\phi\left(a^{2}-1\right)=1$. It remains to show that $\phi\left(a+\sqrt{a^{2}-1}\right)=\phi(2 a+2)$. This follows because

$$
\frac{2 a+2 \sqrt{a^{2}-1}}{a+1}=\left(1+\frac{\sqrt{a^{2}-1}}{a+1}\right)^{2} .
$$

The next theorem generalizes Theorem 2.6; it is the analogue of $K_{n}(x)=\frac{\sqrt{\pi}}{\Gamma\left(n+\frac{1}{2}\right)}\left(\frac{x}{2}\right)^{n} \int_{1}^{\infty} e^{-x w}\left(w^{2}-1\right)^{n-1 / 2} d w \quad[\mathbf{2 0},(5.10 .24)]$

THEOREM 2.7. Unless $N=1$ and $x=0$,

$$
K_{N}(x)=\frac{N(x / 2) G(\phi)}{G(N \phi)} \sum_{w} N \phi\left(w^{2}-1\right) \zeta^{w x} .
$$

Proof. The result is clear for $x=0$, so assume $x \neq 0$. By (2.5),

$$
K_{N}(x) G(N \phi)=\sum_{t, u \neq 0} N(t) N \phi(u) \zeta^{x(t+\bar{t}) / 2+u} .
$$

Replace $t$ by $t x /(2 u)$ to get

$$
K_{N}(x) G(N \phi)=\sum_{t, u \neq 0} \phi(u) N\left(\frac{t x}{2}\right) \zeta^{x(t x / 2 u+2 u / t x) / 2+u}
$$

Now replace $u$ by $u t$ to get

$$
\begin{aligned}
\bar{N}\left(\frac{x}{2}\right) K_{N}(x) G(N \phi) & =\sum_{t, u} \phi(u) N \phi(t) \xi^{u(t+1)+\bar{u} x^{2} / 4} \\
& =\sum_{t} N \phi(t) S(x, t)
\end{aligned}
$$

where

$$
S(x, t):=\sum_{u} \phi(u) \zeta^{u(t+1)+\bar{u} x^{2} / 4} .
$$

If $t+1=0, S(x, t)=G(\phi)$. If $\phi(t+1)=-1$, then replacement of $u$ by $u x^{2} /(4 t+4)$ yields

$$
S(x, t)=\phi(t+1) \sum_{u} \phi(u) \zeta^{\bar{u}(t+1)+u x^{2} / 4}=-S(x, t),
$$


so $S(x, t)=0$. If $\phi(t+1)=1$, then replacement of $u$ by $u x /(2 \sqrt{t+1})$ yields

$$
\begin{aligned}
S(x, t) & =\phi(2 x \sqrt{t+1}) \sum_{u} \phi(u) \zeta^{x \sqrt{t+1}(u+\bar{u}) / 2} \\
& =\phi(2 x \sqrt{t+1}) K_{\phi}(x \sqrt{t+1})=G(\phi)\left(\zeta^{x \sqrt{t+1}}+\zeta^{-x \sqrt{t+1}}\right)
\end{aligned}
$$

by Theorem 2.6. Thus, with $w^{2}=t+1, \bar{N}(x / 2) K_{N}(x) G(N \phi)=$ $G(\phi) \sum_{w} N \phi\left(w^{2}-1\right) \zeta^{x w}$, as desired.

Finally, we record the following well-known special case of the Hasse-Davenport multiplication formula mentioned in the Introduction:

$$
G\left(A^{2}\right) G(\phi)=A(4) G(A) G(A \phi) \text {. }
$$

3. Multivariable Hermite sums. The following theorem is the analogue of Taylor's theorem in several variables. We omit the proof, as it is similar to that of Theorem 2.4. We shall write a for the vector $\left(a_{1}, \ldots, a_{r}\right)$ $\in G F(q)^{r}$ and $\mathbf{N}$ for the vector of characters $\left(N_{1}, \ldots, N_{r}\right)$.

Theorem 3.1. Let $F: G F(q)^{r} \rightarrow \mathbf{C}$ and fix $\mathbf{a} \in G F(q)^{r}$. If $u_{i} \neq a_{i}$ for each $i, 1 \leq i \leq r$, then

$$
F(\mathbf{u})=\left.(q-1)^{-r} \sum_{\mathbf{N}} D_{u_{1}}^{N_{1}} \cdots D_{u_{r}}^{N_{r}} F(\mathbf{u})\right|_{\mathbf{u}=\mathbf{a}} \prod_{i=1}^{r} G\left(\bar{N}_{i}\right) N_{i}\left(a_{\imath}-u_{\imath}\right) .
$$

Moreover, this expansion is unique in the sense that if for all $\mathbf{u}$,

$$
0=\sum_{\mathbf{N}} R(\mathbf{N}) \prod_{i=1}^{r} N_{i}\left(a_{i}-u_{i}\right)
$$

then $R(\mathbf{N})=0$ for all $\mathbf{N}$.

Fix a symmetric $r \times r$ matrix $D$ over $G F(q)$ with nonzero determinant $d \in G F(q)$. Given a row vector $\mathbf{x}$, let $\mathbf{x}^{t}$ denote its transpose. For the rest of this section, let $\mathbf{w}$ and $\mathbf{u}$ be vectors with $w_{i} u_{i} \neq 0,1 \leq i \leq r$. In view of Theorem 3.1 with $\mathbf{a}=0$, we can define multivariable Hermite character sums $G_{\mathbf{N}}(\mathbf{x})$ and $H_{\mathbf{M}}(\mathbf{x})$ by

$$
\begin{aligned}
\zeta^{\left(\left(\mathbf{x}-D^{-1} \mathbf{u}\right)^{t} D\left(\mathbf{x}-D^{-1} \mathbf{u}\right)-\mathbf{x}^{t} D \mathbf{x}\right) / 2} & \\
= & (q-1)^{-r} \sum_{\mathbf{N}} G_{\mathbf{N}}(\mathbf{x}) \prod_{i=1}^{r} G\left(\bar{N}_{i}\right) N_{i}\left(-u_{i}\right),
\end{aligned}
$$

and

$$
\begin{aligned}
& \zeta^{\left((\mathbf{x}-\mathbf{w})^{t} D(\mathbf{x}-\mathbf{w})-\mathbf{x}^{t} D \mathbf{x}\right) / 2} \\
& =(q-1)^{-r} \sum_{\mathbf{M}} H_{\mathbf{M}}(\mathbf{x}) \prod_{i=1}^{r} G\left(\bar{M}_{i}\right) M_{l}\left(-w_{l}\right) .
\end{aligned}
$$


The following theorem is the analogue of the biorthogonality property of Hermite polynomials [6, p. 286, (1)].

THEOREM 3.2.

$$
\begin{aligned}
L(\mathbf{N}, \mathbf{M}): & =\sum_{\mathbf{x}} \zeta^{\mathbf{x}^{t} D \mathbf{x} / 2} G_{\mathbf{N}}(x) H_{\mathbf{M}}(\mathbf{x}) \\
& = \begin{cases}(q-1)^{r} \phi\left(2^{r} d\right) G^{r}(\phi) \prod_{i=1}^{r} \frac{M_{i}(-1)}{G\left(\bar{M}_{i}\right)}, & \text { if } \mathbf{N}=\mathbf{M} \\
0, & \text { otherwise. }\end{cases}
\end{aligned}
$$

Proof. Multiplying the equalities in (3.1) and (3.2), we obtain

$$
\begin{aligned}
(q- & 1)^{-2 r} \sum_{\mathbf{M}, \mathbf{N}} L(\mathbf{M}, \mathbf{N}) \prod_{i=1}^{r} G\left(\bar{M}_{i}\right) G\left(\bar{N}_{i}\right) M_{i}\left(-w_{i}\right) N_{i}\left(-u_{i}\right) \\
& =\zeta^{\left(\mathbf{u}^{t} D^{-1} \mathbf{u}+\mathbf{w}^{t} D \mathbf{w}\right) / 2} \sum_{\mathbf{x}} \zeta^{\left(\mathbf{x}^{t} D \mathbf{x}-2 \mathbf{x}^{t}(\mathbf{u}+D \mathbf{w})\right) / 2} \\
& =\zeta^{\left(\mathbf{u}^{t} D^{-1} \mathbf{u}+\mathbf{w}^{t} D \mathbf{w}\right) / 2} \sum_{\mathbf{x}} \zeta^{\left(\left(\mathbf{x}-\mathbf{w}-D^{-1} \mathbf{u}\right)^{t} D\left(\mathbf{x}-\mathbf{w}-D^{-1} \mathbf{u}\right)-(\mathbf{u}+D \mathbf{w})^{t} D^{-1}(\mathbf{u}+D \mathbf{w})\right) / 2} \\
& =\zeta^{\left(\mathbf{u}^{t} D^{-1} \mathbf{u}+\mathbf{w}^{t} D \mathbf{w}\right) / 2} \sum_{\mathbf{x}} \zeta^{\left(\mathbf{x}^{t} D \mathbf{x}-(\mathbf{u}+D \mathbf{w})^{t} D^{-1}(\mathbf{u}+D \mathbf{w})\right) / 2} \\
& =\zeta^{-\mathbf{w}^{t} \mathbf{u}} \sum_{\mathbf{x}} \zeta^{\mathbf{x}^{t} D \mathbf{x} / 2} .
\end{aligned}
$$

Since $q$ is odd, there exists an invertible matrix $Q$ over $G F(q)$ such that $Q^{t} D Q$ is diagonal [4, p. 253, Theorem 15]. Thus, replacing $\mathbf{x}$ by $Q \mathbf{x}$, we find that

$$
\begin{aligned}
& \zeta^{-\mathbf{w}^{t} \mathbf{u}} \sum_{\mathbf{x}} \zeta^{\mathbf{x}^{t} D \mathbf{x} / 2}=\zeta^{-\mathbf{w}^{t} \mathbf{u}} \phi\left(2^{r} d\right) G^{r}(\phi) \\
& \quad=\phi\left(2^{r} d\right) G^{r}(\phi)(q-1)^{-r} \sum_{\mathbf{M}} \prod_{i=1}^{r} G\left(\bar{M}_{i}\right) M_{i}\left(-w_{i} u_{i}\right),
\end{aligned}
$$

by Theorem 3.1. Comparing coefficients of $\prod_{i=1}^{r} M_{i}\left(-w_{i} u_{i}\right)$, we easily obtain the result.

4. Hermite sums. In this section, we catalogue the theorems (in somewhat arbitrary order) corresponding to what we believe to be the most important classical formulas for Hermite functions. In many cases, it is more difficult to construct an elegant analogue (and find general 
conditions of validity) than it is to give proofs. If for example one had made the reasonable guess that the analogue of the binomial coefficient in (4.29) is

$$
q G(N) /((q-1) G(B) G(N \bar{B}))
$$

(instead of $G(\bar{B}) G(B \bar{N}) /((q-1) G(\bar{N}))$, used in Theorem 4.29), unnecessary complications would have resulted.

Corresponding to the Rodriguez formula

$$
H_{n}(x)=(-1)^{n} e^{x^{2}} \frac{d^{n}}{d x^{n}} e^{-x^{2}} \quad[6, \mathrm{p} .193,(7)],
$$

we have

THEOREM 4.1. $H_{N}(x)=N(-1) \zeta^{-x^{2}} D^{N} \zeta^{x^{2}}$.

Proof. By (2.8) and (2.1),

$$
\begin{aligned}
D^{N} \zeta^{x^{2}} & =\frac{1}{G(\bar{N})} \sum_{u} \bar{N}(u) \zeta^{(x-u)^{2}}=\frac{N(-1)}{G(\bar{N})} \sum_{u} \bar{N}(u) \zeta^{(x+u)^{2}} \\
& =N(-1) \zeta^{x^{2}} H_{N}(x) .
\end{aligned}
$$

Corresponding to

$$
\frac{d^{m}}{d x^{m}} H_{n}(x)=(-2)^{m} \frac{\Gamma(m-n)}{\Gamma(-n)} H_{n-m}(x) \quad[6, \text { p. 119, (15)] }
$$

we have

THEOREM 4.2.

$$
D^{M} H_{N}(x)=\frac{M(-2) G(M \bar{N})}{G(\bar{N})} H_{N \bar{M}}(x) .
$$

Proof.

$$
\begin{aligned}
G(\bar{N}) & D^{M} H_{N}(x)=\frac{G(\bar{N})}{G(\bar{M})} \sum_{t} \bar{M}(t) H_{N}(x-t) \\
& =\frac{1}{G(\bar{M})} \sum_{t, u \neq 0} \bar{M}(t) \bar{N}(u) \zeta^{u^{2}+2 u(x-t)}=\sum_{u} M(-2 u) \bar{N}(u) \zeta^{u^{2}+2 u x} \\
& =M(-2) G(M \bar{N}) H_{N \bar{M}}(x) .
\end{aligned}
$$


Corresponding to

(4.3) $\frac{d^{m}}{d x^{m}} e^{-x^{2}} H_{n}(x)=(-1)^{m} e^{-x^{2}} H_{n+m}(x) \quad[6$, p. 119, (16)],

we have

THEOREM 4.3. If $M N \neq 1$, then

$$
D^{M} \zeta^{x^{2}} H_{N}(x)=M(-1) \zeta^{x^{2}} H_{M N}(x) .
$$

Proof. Since $M N \neq 1, D^{M} D^{N}=D^{M N}$ by Theorem 2.1. Thus, by Theorem 4.1,

$$
\begin{aligned}
D^{M} \zeta^{x^{2}} H_{N}(x) & =N(-1) D^{M} D^{N} \zeta^{x^{2}} \\
& =N(-1) D^{M N} \zeta^{x^{2}}=N(-1) \zeta^{x^{2}} M N(-1) H_{M N}(x) .
\end{aligned}
$$

Corresponding to

$$
H_{0}(x)=1 \quad[6, \text { p. 193, (8) }],
$$

we have

THEOREM 4.4. $H_{N}(x)=1-\zeta^{-x^{2}} G(\phi)$ when $N=1$.

Proof. By Theorem 2.1, $D^{1} \zeta^{x^{2}}=\zeta^{x^{2}}-G(\phi)$, so the result follows from Theorem 4.1. Alternatively, put $N=1$ in (2.1).

Corresponding to

$$
H_{n}(-x)=(-1)^{n} H_{n}(x) \quad[6, \text { p. 193, (14)] }
$$

we have

THEOREM 4.5. $H_{N}(-x)=N(-1) H_{N}(x)$.

Proof. Replace $u$ by $-u$ in (2.1).

Corresponding to

$$
H_{2 m}(0)=(-1)^{m}(2 m) ! / m ! \quad[6, \text { p. 193, (15)] }
$$

we have

THEOREM 4.6.

$$
H_{N}(0)= \begin{cases}0, & \text { if } N \text { is not a square } \\ \frac{G(\bar{M})+G(\bar{M} \phi)}{G\left(\bar{M}^{2}\right)}, & \text { if } N=M^{2}\end{cases}
$$


Proof. $G(\bar{N}) H_{N}(0)=\sum_{u} \bar{N}(u) \zeta^{u^{2}}$, which vanishes when $N$ is not a square. If $N=M^{2}$, then

$$
\begin{aligned}
G(\bar{N}) H_{N}(0) & =\sum_{u} \bar{M}\left(u^{2}\right) \zeta^{u^{2}}=\sum_{u} \bar{M}(u) \zeta^{u}\{1+\phi(u)\} \\
& =G(\bar{M})+G(\bar{M} \phi) .
\end{aligned}
$$

Corresponding to the generating function formula

$$
e^{2 x z-z^{2}}=\sum_{n=0}^{\infty} \frac{H_{n}(x) z^{n}}{n !} \quad[6, \text { p. 194, (19) }]
$$

we have

TheOREM 4.7. For $z \neq 0, \zeta^{z^{2}-2 x z}=\sum_{N} N(-z) G(\bar{N}) H_{N}(x) /(q-1)$.

Proof. By (2.1), the right side above equals

$$
\sum_{N} \frac{N(-z)}{q-1} \sum_{u} \bar{N}(u) \zeta^{u^{2}+2 u x}=\sum_{u=-z} \zeta^{u^{2}+2 u x}=\zeta^{z^{2}-2 z x} .
$$

Corresponding to the polynomial expansion

$$
H_{n}(x)=n ! \sum_{m=0}^{[n / 2]} \frac{(-1)^{m}(2 x)^{n-2 m}}{m !(n-2 m) !} \quad[6, \mathrm{p} .193,(9)],
$$

we have

THEOREM 4.8. For $x \neq 0$,

$$
H_{N}(x)=\frac{1}{G(\bar{N})(q-1)} \sum_{M} N \bar{M}^{2}(2 x) G(\bar{M}) G\left(M^{2} \bar{N}\right) .
$$

Proof. By Theorem 2.4 with $a=0$,

$$
H_{N}(x)=\left.\frac{1}{q-1} \sum_{A} G(\bar{A}) D^{A} H_{N}(x)\right|_{x=0} A(-x) .
$$

By Theorem 4.2,

$$
\left.D^{A} H_{N}(x)\right|_{x=0}=\frac{A(-2) G(A \bar{N})}{G(\bar{N})} H_{N A}(0) .
$$

Thus, by Theorem 4.6,

$$
\left.D^{A} H_{N}(x)\right|_{x=0}= \begin{cases}0, & \text { if } N \bar{A} \text { is not a square } \\ \frac{A(-2)}{G(\bar{N})}(G(\bar{M})+G(\bar{M} \phi)), & \text { if } N \bar{A}=M^{2} .\end{cases}
$$

Replacing $A$ by $N \bar{M}^{2}$ in (4.8a), we obtain the desired result. 
Corresponding to the integral representation

(4.9) $\quad H_{n}(x)=\frac{(-2 i)^{n} e^{x^{2}}}{\sqrt{\pi}} \int_{-\infty}^{\infty} e^{2 i t x-t^{2}} t^{n} d t \quad[\mathbf{2 0},(4.11 .4)]$ we have

THEOREM 4.9. If $N \neq 1$, then

$$
H_{N}(x)=\frac{\zeta^{-x^{2}} G(\phi)}{q} \sum_{u} N(2 u) \zeta^{2 u x-u^{2}}
$$

Proof.

$$
\begin{aligned}
L & :=G(\bar{N}) \zeta^{-x^{2}} G(\phi) q^{-1} \sum_{u} N(2 u) \zeta^{2 u x-u^{2}} \\
& =G(\phi) q^{-1} \sum_{u, t \neq 0} N\left(\frac{2 u}{t}\right) \zeta^{t-(u-x)^{2}} .
\end{aligned}
$$

Replace $t$ by $2 t u$ to obtain

$$
L=G(\phi) q^{-1} \sum_{u \neq 0} \sum_{t} \bar{N}(t) \xi^{2 t u-(u-x)^{2}} .
$$

The condition $u \neq 0$ may be dropped since $N \neq 1$. Replace $u$ by $u+x$ to obtain

$$
\begin{aligned}
L & =G(\phi) q^{-1} \sum_{t} \bar{N}(t) \zeta^{2 t x} \sum_{u} \zeta^{2 t u-u^{2}} \\
& =G(\phi) q^{-1} \sum_{t} \bar{N}(t) \zeta^{2 t x+t^{2}} \phi(-1) G(\phi) \\
& =\sum_{t} \bar{N}(t) \zeta^{2 t x+t^{2}}=G(\bar{N}) H_{N}(x)
\end{aligned}
$$

as desired.

Corollary 4.10. If $N \neq 1$, then

$$
\bar{H}_{N}(x)=\frac{\bar{N}(2) q \zeta^{x^{2}}}{G(\phi) G(N)} H_{N}(x)
$$

where the bar on the left denotes complex conjugation.

Proof. By Theorem 4.9, the right side above equals

$$
\frac{1}{G(N)} \sum_{u} N(u) \zeta^{2 u x-u^{2}}=\frac{N(-1)}{G(N)} \sum_{u} N(u) \zeta^{-u^{2}-2 u x}=\bar{H}_{N}(x) \text {. }
$$


Corresponding to

$$
\int_{-\infty}^{\infty} e^{-x^{2}+a x} H_{n}(x)=\sqrt{\pi} a^{n} e^{a^{2} / 4} \quad[20, \text { p. 74] }
$$

we have

THEOREM 4.11. Unless both $a=0$ and $N=1$,

$$
\sum_{x} \zeta^{x^{2}-2 a x} H_{N}(x)=G(\phi) N(2 a) \zeta^{-a^{2}}
$$

Proof.

$$
\begin{aligned}
G(\bar{N}) \sum_{x} \zeta^{x^{2}-2 a x} & H_{N}(x)=\sum_{x, t} \bar{N}(t) \zeta^{t^{2}+2 x(t-a)+x^{2}} \\
& =\zeta^{-a^{2}} \sum_{t} \bar{N}(t) \zeta^{2 t a} \sum_{x} \zeta^{(x+t-a)^{2}}=\zeta^{-a^{2}} G(\phi) \sum_{t} \bar{N}(t) \zeta^{2 t a}
\end{aligned}
$$

and the result follows.

Corresponding to the integral equation

$$
\text { (4.12) } \quad H_{n}(x)=\frac{e^{x^{2} / 2}}{i^{n} \sqrt{2 \pi}} \int_{-\infty}^{\infty} e^{i x y-y^{2} / 2} H_{n}(y) d y \quad[\mathbf{2 0},(4.12 .3)]
$$

we have

THEOREM 4.12. If $N \neq 1$, then

$$
H_{N}(x)=\frac{\phi(2) G(\phi) N(-1)}{q} \zeta^{-x^{2} / 2} \sum_{u} \zeta^{-x u-u^{2} / 2} \bar{H}_{\bar{N}}(u) .
$$

Proof. By Theorem 4.9, the right side above equals

$$
\begin{gathered}
\frac{\phi(2) G(\phi)}{q} \overline{G(\phi)} q^{-1} \sum_{u} \zeta^{-x u+\left(u^{2}-x^{2}\right) / 2} \sum_{t} N(-2 t) \zeta^{t^{2}-2 t u} \\
=\phi(2) q^{-1} \sum_{t} N(-2 t) \zeta^{t^{2}-x^{2} / 2} \sum_{u} \zeta^{\left(u^{2}-2 x u-4 t u\right) / 2} \\
=q^{-1} G(\phi) \sum_{t} N(-2 t) \zeta^{t^{2}-x^{2} / 2-(x+2 t)^{2} / 2} \\
=\zeta^{-x^{2}} G(\phi) q^{-1} \sum_{t} N(-2 t) \zeta^{-t^{2}-2 t x}=H_{N}(x)
\end{gathered}
$$

again by Theorem 4.9 . 
Corresponding to

$$
H_{2 n}(x)=(-4)^{n} n ! L_{n}^{-1 / 2}\left(x^{2}\right) \quad[6, \text { p. } 193,(2)],
$$

we have

THEOREM 4.13. If $x \neq 0$ and $N \neq \phi$, then

$$
H_{N^{2}}(x)=\frac{q N(4)}{G(\bar{N})} L_{N}^{\phi}\left(x^{2}\right) .
$$

Proof. By Theorem 4.8,

$$
H_{N^{2}}(x)=\frac{1}{(q-1) G\left(\bar{N}^{2}\right)} \sum_{M} N^{2} \bar{M}^{2}(2 x) G(\bar{M}) G\left(M^{2} \bar{N}^{2}\right) .
$$

Replace $M$ by $N \bar{M}$ to obtain

$$
\text { (4.13a) } H_{N^{2}}(x)=\frac{1}{(q-1) G\left(\bar{N}^{2}\right)} \sum_{M} M^{2}(2 x) G(M \bar{N}) G\left(\bar{M}^{2}\right) \text {. }
$$

By Theorem 2.4, for $z \neq 0$,

$$
\begin{aligned}
q L_{N}^{A}(z) & =\left.\sum_{M} \frac{G(\bar{M})}{q-1} D^{M} q L_{N}^{A}(z)\right|_{z=0} M(-z) \\
& =\sum_{M} \frac{M(-z)}{q-1} \sum_{t} \bar{M}(t) q L_{N}^{A}(-t) .
\end{aligned}
$$

Thus, by the definition (2.2) of $L_{N}^{A}$,

$$
\begin{aligned}
q L_{N}^{A}(z) & =\sum_{M} \frac{M(-z)}{q-1} \sum_{t} \bar{M}(t) \sum_{u} N A(1+u) \bar{N}(u) \zeta^{-t u} \\
& =\sum_{M} \frac{M(z)}{q-1} G(\bar{M}) \sum_{u} M \bar{N}(u) N A(1+u) \\
& =\frac{N(-1)}{q-1} \sum_{M} M(-z) G(\bar{M}) J(M \bar{N}, N A)
\end{aligned}
$$

Therefore, with $A=\phi$ and $z=x^{2}$,

(4.13b) $\frac{N(4)}{G(\widetilde{N})} q L_{N}^{\phi}\left(x^{2}\right)$

$$
=\frac{N(-4)}{G(\bar{N})(q-1)} \sum_{M} M\left(-x^{2}\right) G(\bar{M}) J(M \bar{N}, N \phi) .
$$

Comparing (4.13a) and (4.13b), we see that it remains to show that

$$
\frac{M(4) G(M \bar{N}) G\left(\bar{M}^{2}\right)}{G\left(\bar{N}^{2}\right)}=\frac{N(-4) M(-1) G(\bar{M}) J(M \bar{N}, N \phi)}{G(\bar{N})}
$$


If $M=\phi$, then since $N \neq \phi$, we have $J(M \bar{N}, N \phi)=-N \phi(-1)$, so (4.13c) follows from (2.15) with $A=\bar{N}$. If $M \neq \phi$, then $J(M \bar{N}, N \phi)=$ $G(M \bar{N}) G(N \phi) / G(M \phi)$, and again (4.13c) follows from (2.15).

Corresponding to

$$
H_{-1 / 2}(z)=e^{z^{2} / 2} \sqrt{\frac{z}{2 \pi}} K_{1 / 4}\left(\frac{z^{2}}{2}\right)
$$

(which is stated incorrectly in both [6, p. 119, (20)] and [20, p. 298, \#6]), we have

THEOREM 4.14. Let $x \neq 0$ and let $N$ be quartic, so that $N^{4}=1$. Then

$$
H_{\phi}(x)=\frac{\zeta^{-x^{2} / 2} \phi(2 x)}{G(\phi)} K_{N}\left(\frac{x^{2}}{2}\right) .
$$

Proof. By Theorem 4.13 and the definition of $L_{N}^{\phi}$,

$$
\begin{aligned}
H_{\phi}(x) & =H_{N^{2}}(x)=\frac{q N(4)}{G(\bar{N})} L_{N}^{\phi}\left(x^{2}\right) \\
& =\frac{N(4)}{G(\bar{N})} \sum_{t} \bar{N}(t) N \phi(1+t) \zeta^{t x^{2}}=\frac{\phi(2)}{G(\bar{N})} \sum_{t} \bar{N}\left(t+t^{2}\right) \zeta^{t x^{2}} \\
& =\frac{\phi(2)}{G(\bar{N})} \sum_{t} \bar{N}\left(t^{2}-\overline{4}\right) \zeta^{(t-\overline{2}) x^{2}}=\frac{\zeta^{-x^{2} / 2}}{G(\bar{N})} \sum_{t} \bar{N}\left(t^{2}-1\right) \zeta^{t x^{2} / 2} .
\end{aligned}
$$

Thus, by Theorem 2.7,

$$
H_{\phi}(x)=\frac{\zeta^{-x^{2} / 2}}{G(\bar{N})} \frac{G(\bar{N}) \bar{N}\left(x^{2} / 4\right)}{G(\phi)} K_{N}\left(\frac{x^{2}}{2}\right)
$$

as desired.

Corresponding to

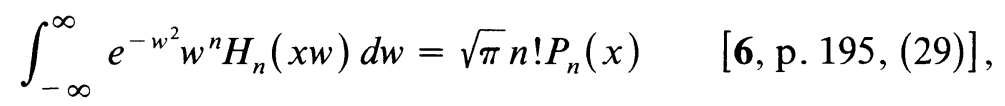

we have

THEOREM 4.15. Let $N \neq 1$. Then

$$
\sum_{w} \zeta^{w^{2}} N(w) H_{N}(x w)=\frac{q N(-1) G(\phi)}{G(\bar{N})} P_{N}(x)+\frac{q N(-1)}{G(\bar{N})} \sum_{\substack{w \\ w^{2}+1=2 x w}} N(w) .
$$

(Note that the last sum vanishes if $\phi\left(x^{2}-1\right)=-1$.) 
Proof. By (2.3),

$$
\begin{array}{rl}
G(\bar{N}) \sum_{w} \zeta^{w^{2}} & N(w) H_{N}(x w)=\sum_{u} \bar{N}(u) \zeta^{u^{2}\left(1-x^{2}\right)} \sum_{w} N(w) \zeta^{(w+u x)^{2}} \\
& =\sum_{u \neq 0} \zeta^{u^{2}\left(1-x^{2}\right)} \sum_{w} N(w) \zeta^{(w u+u x)^{2}}=\sum_{w} N(w) \sum_{u} \zeta^{u^{2}\left(1+w^{2}+2 w x\right)} \\
& =\sum_{w} N(w) \phi\left(1+w^{2}+2 w x\right) G(\phi)+q \sum_{w} N(w) \\
& =q N(-1) G(\phi) P_{N}(x)+q N(-1) \sum_{\substack{w \\
w^{2}+1=-2 w x}} N(w) .
\end{array}
$$

Corresponding to the addition theorem

$$
H_{n}(a x+b y)=\sum_{m=0}^{n}\left(\begin{array}{c}
n \\
m
\end{array}\right) a^{m} b^{n-m} H_{m}(x) H_{n-m}(y)
$$

we have

THEOREM 4.16. If $a, b, x, y \in G F(q)$ with $a^{2}+b^{2}=1$ and $a b \neq 0$, then

$$
H_{N}(a x+b y)=\sum_{M} \frac{G(\bar{M}) G(M \bar{N})}{(q-1) G(\bar{N})} M(a) N \bar{M}(b) H_{M}(x) H_{N \bar{M}}(y) .
$$

Proof. Let $w \neq 0$. By Theorem 4.7,

$$
\zeta^{a^{2} w^{2}+2 w a x}=\sum_{M} \frac{M(a w) G(\bar{M})}{q-1} H_{M}(x)
$$

and

$$
\zeta^{b^{2} w^{2}+2 w b y}=\sum_{A} \frac{A(b w) G(\bar{A})}{q-1} H_{A}(y) .
$$

Multiply to obtain

(4.16a) $\zeta^{w^{2}+2 w(a x+b y)}$

$$
=(q-1)^{-2} \sum_{A, M} M(a) A(b) M A(w) G(\bar{M}) G(\bar{A}) H_{M}(x) H_{A}(y) .
$$

Also, by Theorem 4.7,

$$
\zeta^{w^{2}+2 w(a x+b v)}=\sum_{N} \frac{G(\bar{N})}{q-1} N(w) H_{N}(a x+b y) .
$$


The result now follows from Theorem 2.4 upon comparing the coefficients of $N(w)$ in (4.16a) and (4.16b).

Corresponding to (cf. (4.15))

$$
\int_{-\infty}^{\infty} e^{-a w^{2}} H_{2 m}(w) d w=\frac{\sqrt{\pi}(2 m) !}{m !} a^{-1 / 2}\left(a^{-1}-1\right)^{m}
$$

$[20$, p. 75],

we have

THEOREM 4.17. Let $a \neq 0,1$. Then

$\sum_{w} \zeta^{a w^{2}} H_{N}(w)$

$$
=\left\{\begin{array}{r}
0, \quad \text { if } N \text { is not a square } \\
\frac{G(\phi)}{G\left(\bar{M}^{2}\right)} \phi(a)\left(M\left(1-a^{-1}\right) G(\bar{M})+M \phi\left(1-a^{-1}\right) G(\bar{M} \phi)\right), \\
\text { if } N=M^{2} .
\end{array}\right.
$$

Proof. Let $x=a^{-1}$. Then

$$
\begin{aligned}
G(\bar{N}) & \sum_{w} \zeta^{a w^{2}} H_{N}(w)=\sum_{w} \zeta^{w^{2} a} \sum_{u} \bar{N}(u) \zeta^{u^{2}+2 u w} \\
= & \sum_{u} \bar{N}(u) \zeta u^{u^{2}(1-x)} \sum_{w} \zeta^{a(w+u x)^{2}}=\phi(a) G(\phi) \sum_{u} \bar{N}(u) \zeta^{u^{2}(1-x)},
\end{aligned}
$$

and the result easily follows.

A generalization of the formula

$$
\int_{0}^{\infty} e^{-x^{2}} H_{n}(x)^{2} \cos (x y \sqrt{2}) d x=e^{-y^{2} / 2} \sqrt{\pi} 2^{n-1} n ! L_{n}^{0}\left(y^{2}\right),
$$

which is incorrectly stated in [6, p.195, (33)], is

$$
\begin{aligned}
\int_{-\infty}^{\infty} e^{-x^{2}-2 i x y} H_{n}(x) H_{m}(x) d x & \\
& =L_{m}^{n-m}\left(2 y^{2}\right) e^{-y^{2}}(-i y)^{n-m} 2^{n} \sqrt{\pi} m !,
\end{aligned}
$$

which is stated incorrectly in [22, (4.166)]. Corresponding to (4.18), we have (cf. Theorem 4.11)

THEOREM 4.18. Let $N \neq 1, a \neq 0$. Then

$$
L:=\sum_{x} \zeta^{x^{2}+2 x a} H_{N}(x) H_{M}(x)=\frac{\zeta^{-a^{2}} q G(\phi) N(-2 a) \bar{M}(a)}{G(\bar{M})} L_{M}^{N \bar{M}}\left(-2 a^{2}\right) \text {. }
$$


Proof. By Theorems 2.3 and 4.1,

$$
L=\sum_{x} H_{M}(x) \zeta^{2 a x}\left(N(-1) D^{N} \zeta^{x^{2}}\right)=\sum_{x} \zeta^{x^{2}} D^{N}\left(\zeta^{2 a x} H_{M}(x)\right) .
$$

Thus,

$$
\begin{aligned}
L & =\frac{1}{G(\bar{N})} \sum_{x} \zeta^{x^{2}} \sum_{t} \bar{N}(t) \zeta^{2 a(x-t)} H_{M}(x-t) \\
& =\frac{1}{G(\bar{N}) G(\bar{M})} \sum_{s, t} \bar{N}(t) \bar{M}(s) \zeta^{s^{2}-2 s t-2 a t} \sum_{x} \zeta^{x^{2}+2 x(a+s)} \\
& =\frac{G(\phi)}{G(\bar{N}) G(\bar{M})} \sum_{s, t} \bar{N}(t) \bar{M}(s) \zeta^{s^{2}-2 s t-2 a t-(a+s)^{2}} \\
& =\frac{G(\phi) \zeta^{-a^{2}}}{G(\bar{N}) G(\bar{M})} \sum_{s, t} \bar{N}(t) \bar{M}(s) \zeta^{-2(s t+a t+a s)}
\end{aligned}
$$

Since $N \neq 1$, the term with $s=-a$ may be excluded. Replace $t$ by $-t /(a+s)$ to get

$$
\begin{aligned}
L & =\frac{G(\phi) \zeta^{-a^{2}}}{G(\bar{N}) G(\bar{M})} \sum_{s, t} N(-a-s) \bar{N}(t) \bar{M}(s) \zeta^{2 t-2 a s} \\
& =\frac{G(\phi) \zeta^{-a^{2}} N(-2)}{G(\bar{M})} \sum_{s} N(a+s) \bar{M}(s) \zeta^{-2 a s}
\end{aligned}
$$

Since $a \neq 0$,

$$
L=\frac{G(\phi) \zeta^{-a^{2}} N(-2 a) \bar{M}(a)}{G(\bar{M})} \sum_{s} N(s+1) \bar{M}(s) \zeta^{-2 a^{2} s}
$$

and the result follows by the definition of $L_{N}^{A}(x)$.

Corresponding to the orthogonality relation

$$
\begin{aligned}
\int_{-\infty}^{\infty} e^{-x^{2}} H_{m}(x) H_{n}(x) d x \\
\quad= \begin{cases}0, & \text { if } m \neq n \\
2^{n} n ! \sqrt{\pi}, & \text { if } m=n[\mathbf{2 0},(4.13 .1),(4.13 .4)]\end{cases}
\end{aligned}
$$

we have

THEOREM 4.19.

$$
\sum_{x} \zeta^{x^{2}} H_{M}(x) H_{N}(x)= \begin{cases}0, & \text { if } M \neq N \\ \frac{N(-2) G(\phi)(q-1)}{G(\bar{N})}, & \text { if } M=N .\end{cases}
$$


Proof. By (4.18a) with $a=0$,

$$
\sum_{x} \zeta^{x^{2}} H_{M}(x) H_{N}(x)=\frac{G(\phi) N(-2)}{G(\bar{M})} \sum_{s} \bar{M} N(s)
$$

and the result follows.

Corresponding to the Gauss transform formula (4.20) $(2 \pi u)^{-1 / 2} \int_{-\infty}^{\infty} H_{n}(v) \exp \left(-(x-v)^{2} / 2 u\right) d v$

$$
=\left\{\begin{aligned}
(2 x)^{n}, & \text { if } u=1 / 2 \\
(1-2 u)^{n / 2} H_{n}(x / \sqrt{1-2 u}), & \\
& \text { if } 0 \leq u<1 / 2[6, \text { p. 195, (30), (31)] }
\end{aligned}\right.
$$

we have

TheOrem 4.20. Let $N \neq 1, u \neq 0$, and assume $1-2 u=a^{2} \in G F(q)$. Then

$$
L:=\frac{\phi(2 u)}{G(\phi)} \sum_{v} H_{N}(v) \zeta^{(x-v)^{2} / 2 u}= \begin{cases}N(2 x), & \text { if } a=0 \\ N(a) H_{N}(x / a), & \text { if } a \neq 0\end{cases}
$$

Proof.

$$
\begin{aligned}
L & =\frac{\phi(2 u)}{G(\bar{N}) G(\phi)} \sum_{v} \zeta^{(x-v)^{2} / 2 u} \sum_{w} \bar{N}(w) \zeta w^{w^{2}+2 w v} \\
& =\frac{\phi(2 u)}{G(\bar{N}) G(\phi)} \sum_{w} \bar{N}(w) \zeta^{w^{2}+2 w x} \sum_{s} \zeta^{2 w s+s^{2} / 2 u},
\end{aligned}
$$

where we have replaced $v$ by $s+x$. Now,

$$
\sum_{s} \zeta^{2 w s+s^{2} / 2 u}=\zeta^{-2 w^{2} u} \phi(2 u) G(\phi)
$$

Therefore,

$$
L=\frac{1}{G(\bar{N})} \sum_{w} \bar{N}(w) \zeta^{w^{2} a^{2}+2 w x} .
$$

If $a=0$, clearly $L=N(2 x)$. Suppose $a \neq 0$. Then

$$
L=\frac{N(a)}{G(\bar{N})} \sum_{w} \bar{N}(w) \zeta^{w^{2}+2 \lambda w / a}=N(a) H_{N}(x / a) .
$$

Corresponding to

(4.21) $H_{n}(x) H_{-n-1}(x)=e^{x^{2}} \int_{0}^{\infty} J_{n+1 / 2}\left(\frac{t^{2}}{2}\right) \cos \left(x t-\frac{\pi n}{2}\right) e^{-x t} d t$ [6, p. 120, (7)], 
we have

Theorem 4.21. Let $N \neq 1$ and $x \neq 0$. Then

$$
H_{N}(x) H_{\bar{N}}(x)=\frac{\zeta^{-r^{2}} \phi(2) N(-1)}{q} \sum_{u} K_{N \phi}\left(u^{2}\right) \zeta^{2 \times u} .
$$

Proof.

$$
\begin{aligned}
L & :=H_{N}(x) H_{\bar{N}}(x) q N(-1)=H_{N}(x) H_{\bar{N}}(x) G(N) G(\bar{N}) \\
& =\sum_{t, u \neq 0} \bar{N}(u) N(t) \zeta^{t^{2}+u^{2}+2 x(t+u)} .
\end{aligned}
$$

Since $N \neq 1$, we obtain, upon replacing $t$ by $t u$,

$$
\begin{aligned}
L & =\sum_{t} N(t) \sum_{u} \zeta^{u^{2}\left(t^{2}+1\right)+2 x u(t+1)} \\
& =\sum_{t} N(t) \zeta^{-r^{2}(t+1)^{2} /\left(t^{2}+1\right)} \phi\left(t^{2}+1\right) G(\phi) \\
& =\zeta^{-r^{2}} G(\phi) \sum_{t} N(t) \phi\left(t^{2}+1\right) \zeta^{-2 t r^{2} /\left(t^{2}+1\right)} \\
& =\zeta^{-r^{2}} G(\phi) \sum_{t} N \phi(t) \phi(t+\bar{t}) \zeta^{-2 r^{2} /(t+\bar{t})} .
\end{aligned}
$$

It remains to show that

(4.21a) $\sum_{u} K_{N \phi}\left(u^{2}\right) \zeta^{2 \backslash u}=\phi(2) G(\phi) \sum_{t} N \phi(t) \phi(t+\bar{t}) \xi^{-2 x^{2} /(t+\bar{t})}$.

By (2.5),

$$
K_{N \phi}\left(u^{2}\right)=\sum_{t} N \phi(t) \zeta^{u^{2}(t+i) / 2}
$$

so

$$
\sum_{u} K_{N \phi}\left(u^{2}\right) \zeta^{2 x u}=\sum_{t} N \phi(t) \sum_{u} \zeta^{2 x u+u^{2}(t+i) / 2}
$$

Since the rightmost sum on $u$ equals

$$
\zeta^{-2 \imath^{2} /(t+\bar{t})} \phi(t+\bar{t}) \phi(2) G(\phi),
$$

(4.21a) follows. 
Corresponding to

$$
\text { (4.22) } \begin{aligned}
H_{n}\left(\frac{z(1+i)}{2}\right) H_{n}\left(\frac{z(1-i)}{2}\right) \\
=\frac{2^{n+3 / 2}}{\sqrt{\pi} \Gamma(-n)} \int_{0}^{\infty} K_{n+1 / 2}\left(t^{2}\right) \cos \left(z t-\frac{\pi n}{2}\right) e^{-z t} d t
\end{aligned}
$$

we have

THEOREM 4.22. If $N \neq 1$ and $x \neq 0$, then

$$
H_{N}(x) \bar{H}_{\bar{N}}(x)=\frac{\phi N(2) G(N)}{q G(\phi)} \sum_{t} K_{N \phi}\left(t^{2}\right) \zeta^{2 x t}
$$

Proof. This follows from Theorem 4.21 and Corollary 4.10.

Corresponding to (cf. (4.15))

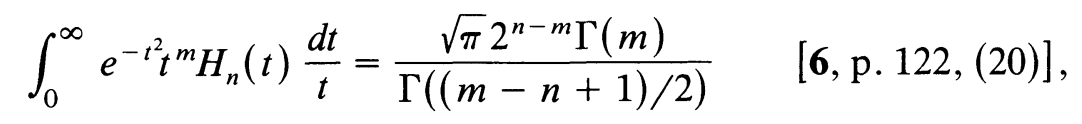

we have

THEOREM 4.23. If $N \neq M$,

$\sum_{t} \zeta^{t^{2}} M(t) H_{N}(t)= \begin{cases}0, & \text { if } M \bar{N} \text { is not a square } \\ \frac{G(M)}{G(M \bar{N})}(G(A)+G(A \phi)), & \text { if } M \bar{N}=A^{2}\end{cases}$

Proof.

$$
\begin{aligned}
\sum_{t} \zeta^{t^{2}} M(t) H_{N}(t) & =\frac{1}{G(\bar{N})} \sum_{t, w} \bar{N}(w) M(t) \zeta^{w^{2}+2 t w+t^{2}} \\
& =\frac{1}{G(\bar{N})} \sum_{t, w \neq 0} M(t) \bar{N}(w-t) \zeta^{w^{2}} \\
& =\frac{1}{G(\bar{N})} \sum_{w \neq 0} M \bar{N}(-w) \zeta^{w^{2}} \sum_{t} M(t) \bar{N}(1-t) \\
& =\frac{J(M, \bar{N})}{G(\bar{N})} \sum_{w} M \bar{N}(w) \zeta^{w^{2}}=\frac{G(M)}{G(M \bar{N})} \sum_{w} M \bar{N}(w) \zeta^{w^{2}},
\end{aligned}
$$

and the result follows. 
By [27, p. 564, (14)], [26, Problem 87], we have

$$
\begin{aligned}
\int_{-\infty}^{\infty} e^{-x^{2}} H_{a}(x) H_{b}(x) H_{c}(x) d x \\
=\frac{\sqrt{\pi} 2^{-n} a ! b ! c !}{(-n-a) !(-n-b) !(-n-c) !},
\end{aligned}
$$

when $n=-(a+b+c) / 2$ is an integer and $a, b, c$ are positive integers. Corresponding to (4.24) is (cf. Theorem 4.19)

THEOREM 4.24.

$$
\begin{aligned}
L:= & \sum_{x} \zeta^{x^{2}} H_{A}(x) H_{B}(x) H_{C}(x) \\
= & \left\{\begin{array}{c}
0, \quad \text { if } A B C \text { is not a square } \\
\frac{G(\phi)}{G(\bar{A}) G(\bar{B}) G(\bar{C})}(\bar{N}(-2) G(A N) G(B N) G(C N) \\
+\bar{N} \phi(-2) G(A N \phi) G(B N \phi) G(C N \phi)), \text { if } \overline{A B C}=N^{2} .
\end{array}\right.
\end{aligned}
$$

Proof. Successively applying Theorems 4.1, 2.3, 2.2, and 4.2, we have

$$
\begin{aligned}
L & =\sum_{x} H_{A}(x) H_{B}(x) C(-1) D^{C} \zeta x^{2}=\sum_{x} \zeta^{x^{2}} D^{C} H_{A}(x) H_{B}(x) \\
& =\sum_{x} \zeta^{x^{2}} \sum_{M} \frac{G(\bar{M}) G(M \bar{C})}{(q-1) G(\bar{C})} D^{M} H_{A}(x) D^{C \bar{M}} H_{B}(x) \\
& =\sum_{M} \frac{G(\bar{M}) G(M \bar{C})}{(q-1) G(\bar{C})} \sum_{x} \zeta^{x^{2}} \frac{C(-2) G(M \bar{A}) G(C \bar{M} \bar{B})}{G(\bar{A}) G(\bar{B})} H_{A \bar{M}}(x) H_{B M \bar{C}}(x) .
\end{aligned}
$$

By Theorem 4.19, $L=0$ if $A B C$ is not a square, while if $A B C=\bar{N}^{2}$, only $M=A C N$ and $M=A C N \phi$ contribute to the sum; these contributions are easily seen to be respectively the two required terms in Theorem 4.24.

Corresponding to Mehler's formula

we have

$$
\begin{aligned}
\left(1-u^{2}\right)^{-1 / 2} \exp \left\{\frac{2 x y u-\left(x^{2}+y^{2}\right) u^{2}}{1-u^{2}}\right\} & \\
= & \sum_{n=0}^{\infty} \frac{H_{n}(x) H_{n}(y)(u / 2)^{n}}{n !} \quad[6, \text { p. 194, (22)], }
\end{aligned}
$$

THEOREM 4.25. Let

$$
r(x, y, z)= \begin{cases}G(\phi) \xi^{-x^{2}}, & \text { if } x=y z \text { and } z= \pm 1 \\ 0, & \text { otherwise. }\end{cases}
$$


Then

$$
\begin{aligned}
L & :=\sum_{N} \frac{G(\bar{N})}{q-1} H_{N}(x) H_{N}(y) \bar{N}(-2 z) \\
& =\phi\left(z^{2}-1\right) \zeta^{\left(2 x y z-x^{2}-y^{2}\right) /\left(1-z^{2}\right)}-\phi(-1) \zeta^{-x^{2}-y^{2}}+r(x, y, z) .
\end{aligned}
$$

(The first term on the right is interpreted as 0 when $z^{2}=1$.)

Proof. The result is clear for $z=0$, so assume $z \neq 0$. Then

$$
\begin{aligned}
L= & \frac{1}{(q-1)} \sum_{N} \frac{\bar{N}(-2 z)}{G(\bar{N})} \sum_{s, t \neq 0} \bar{N}(s t) \xi^{s^{2}+t^{2}+2 s x+2 t y} \\
= & \frac{1}{q(q-1)} \sum_{N \neq 1} \sum_{s, t, u \neq 0} \bar{N}(2 z s t / u) \zeta^{s^{2}+t^{2}+u+2 s x+t y} \\
& -\frac{1}{q-1} \sum_{s, t \neq 0} \zeta^{s^{2}+t^{2}+2 s x+2 t y} \\
= & \frac{1}{q(q-1)} \sum_{N} \sum_{s, t, u \neq 0} \bar{N}(2 z s t / u) \zeta^{s^{2}+t^{2}+u+2 s x+2 t y} \\
& -\frac{1}{q} \sum_{s, t \neq 0} \zeta^{s^{2}+t^{2}+2 s x+2 t y} \\
= & \frac{1}{q} \sum_{s, t \neq 0}\left(\zeta^{s^{2}+t^{2}+2 z s t+2 s x+2 t y}-\zeta^{s^{2}+t^{2}+2 s x+2 t y}\right) .
\end{aligned}
$$

The restrictions $s \neq 0, t \neq 0$ can now be dropped, so

$$
\begin{aligned}
L & =\frac{-G^{2}(\phi)}{q} \zeta^{-x^{2}-y^{2}}+\frac{1}{q} \sum_{s} \zeta^{s^{2}+2 s x-(y+s z)^{2}} \sum_{t} \zeta^{t^{2}+t(2 y+2 z s)+(y+s z)^{2}} \\
& =-\phi(-1) \zeta^{-x^{2}-y^{2}}+\frac{G(\phi)}{q} \zeta^{-y^{2}} \sum_{s} \zeta^{s^{2}\left(1-z^{2}\right)+2 s(x-y z)} .
\end{aligned}
$$

If $z^{2}=1$ and $x=y z$, then

$$
L=-\phi(-1) \zeta^{-x^{2}-y^{2}}+G(\phi) \zeta^{-x^{2}},
$$

as desired. If $z^{2}=1$ and $x \neq y z$, then

$$
L=-\phi(-1) \zeta^{-x^{2}-y^{2}}
$$


as desired. Finally, suppose $z^{2} \neq 1$. Then

$$
\begin{aligned}
L & =-\phi(-1) \zeta^{-x^{2}-y^{2}}+\frac{G(\phi)}{q} \zeta^{-y^{2}-(x-y z)^{2} /\left(1-z^{2}\right)} \phi\left(1-z^{2}\right) G(\phi) \\
& =-\phi(-1) \zeta^{-x^{2}-y^{2}}+\phi\left(z^{2}-1\right) \zeta^{\left(2 x y z-x^{2}-y^{2}\right) /\left(1-z^{2}\right)}
\end{aligned}
$$

as desired.

COROllary 4.26. If $N \neq 1$, and

$$
F(x, y)= \begin{cases}N(-2 x / y), & \text { if } x= \pm y \neq 0 \\ N(2)+N(-2), & \text { if } x=y=0 \\ 0, & \text { otherwise }\end{cases}
$$

then

$$
\begin{aligned}
H_{N}(x) H_{N}(y)= & \frac{F(x, y) G(\phi) \zeta^{-x^{2}}}{G(\bar{N})} \\
& +\frac{N(2)}{G(\bar{N})} \sum_{t} \bar{N}(t) \phi\left(1-t^{2}\right) \zeta^{\left(2 x y t+t^{2} x^{2}+t^{2} y^{2}\right) /\left(1-t^{2}\right)}
\end{aligned}
$$

Proof. By Theorems 4.25 and 2.4,

$$
\begin{aligned}
& H_{N}(x) H_{N}(y) \bar{N}(2) \\
& =D_{z}^{N}\left\{\phi\left(1-z^{2}\right)\right\}^{\left(2 x y z-x^{2} z^{2}-y^{2} z^{2}\right) /\left(z^{2}-1\right)} \\
& \left.-\phi(-1) \zeta^{-x^{2}-y^{2}}+r(x, y, z)\right\}\left.\right|_{z=0} \\
& =\frac{1}{G(\bar{N})} \sum_{t} \bar{N}(t) \phi\left(1-t^{2}\right) \zeta^{\left(2 x y t+x^{2} t^{2}+y^{2} t^{2}\right) /\left(1-t^{2}\right)} \\
& +\frac{1}{G(\bar{N})} \sum_{t} \bar{N}(t) r(x, y,-t),
\end{aligned}
$$

and the result follows since

$$
N(2) \sum_{t} \bar{N}(t) r(x, y,-t)=F(x, y) G(\phi) \zeta^{-x^{2}} .
$$

Under certain conditions [20, p. 71, Theorem 2], a function $f(x)$ has an expansion of the form

$$
f(x)=\sum_{n=0}^{\infty} e_{n} H_{n}(x)
$$


where

$$
e_{n}=\frac{1}{2^{n} n ! \sqrt{\pi}} \int_{-\infty}^{\infty} e^{-x^{2}} f(x) H_{n}(x) d x .
$$

Corresponding to (4.27), we have

ThEOREM 4.27. Let $F: G F(q) \rightarrow$ C. Then

$$
F(u)=\sum_{N} e_{N} H_{N}(u)+q^{-1} \zeta^{-u^{2}} G(\phi) \sum_{t} F(t),
$$

where

$$
e_{N}=\frac{G(\bar{N}) \bar{N}(-2)}{G(\phi)(q-1)} \sum_{x} \zeta^{x^{2}} F(x) H_{N}(x)
$$

Proof. By Theorem 4.25 with $z=1$,

$$
\begin{aligned}
\sum_{N} e_{N} H_{N}(u) & =\frac{1}{G(\phi)} \sum_{x} \zeta^{x^{2}} F(x)\left\{-\phi(-1) \zeta^{x^{2}-u^{2}}+r(x, u, 1)\right\} \\
& =\frac{-\phi(-1)}{G(\phi)} \zeta^{-u^{2}} \sum_{x} F(x)+F(u) .
\end{aligned}
$$

Corresponding to

$$
H_{b}(x) H_{c}(x)=\sum_{m=0}^{\infty} 2^{m} m !\left(\begin{array}{c}
b \\
m
\end{array}\right)\left(\begin{array}{c}
c \\
m
\end{array}\right) H_{b+c-2 m}(x)
$$

$[6$, p. $195,(37)]$

we have

THEOREM 4.28. Let

$$
g(B, C)= \begin{cases}0, & \text { if } B C \text { is not a square } \\ W(2) G(\bar{W})+\phi W(2) G(\bar{W} \phi), & \text { if } B C=W^{2} .\end{cases}
$$

Then

$$
\begin{aligned}
H_{B}(x) H_{C}(x)= & \frac{C(-1) \zeta^{-x^{2}} G(\phi) g(B, C)}{G(\bar{B}) G(\bar{C})}+\frac{1}{G(\bar{B}) G(\bar{C})(q-1)} \\
& \times \sum_{M} M(-2) G(\bar{M}) G(M \bar{B}) G(M \bar{C}) H_{B C \bar{M}^{2}}(x)
\end{aligned}
$$


Proof. By Theorem 4.27 with $F(x)=H_{B}(x) H_{C}(x)$,

(4.28a) $H_{B}(x) H_{C}(x)=\sum_{A} e_{A} H_{A}(x)+q^{-1} \zeta^{-x^{2}} G(\phi) \sum_{t} H_{B}(t) H_{C}(t)$,

where

(4.28b)

$$
e_{A}=\frac{G(\bar{A}) \bar{A}(-2)}{G(\phi)(q-1)} \sum_{x} \zeta^{x^{2}} H_{A}(x) H_{B}(x) H_{C}(x) .
$$

When $A B C$ is a square, then as in Theorem 4.24, let $N^{2}=\bar{A} \bar{B} \bar{C}$, and put $M=N B C$, so that $A=B C \bar{M}^{2}$. Then by (4.28b) and Theorem 4.24,

$$
\begin{aligned}
\sum_{A} e_{A} H_{A}(x) & \\
& =\frac{1}{G(\bar{B}) G(\bar{C})(q-1)} \sum_{M} M(-2) G(\bar{M}) G(M \bar{B}) G(M \bar{C}) H_{B C \bar{M}^{2}}(x) .
\end{aligned}
$$

It is easily checked that

$$
\sum_{t} H_{B}(t) H_{C}(t)=\frac{q C(-1)}{G(\bar{B}) G(\bar{C})} g(B, C),
$$

so the result follows from (4.28a).

The formula

$$
H_{n}(x+a)=\sum_{k=0}^{n}\left(\begin{array}{l}
n \\
k
\end{array}\right)(2 a)^{k} H_{n-k}(x)
$$

is stated in [25, p. 252, Ex. 1] and, in slightly different form, in [25, p. 253, Ex. 8]. Miller [22, p. 106] states (4.29) incorrectly. Corresponding to (4.29), we have

THEOREM 4.29. Let $a \neq 0$. Then

$$
H_{N}(x+a)=\frac{1}{q-1} \sum_{B} \frac{G(\bar{B}) G(B \bar{N})}{G(\bar{N})} B(2 a) H_{N \bar{B}}(x) .
$$

Proof. The right side above equals

$$
\begin{aligned}
& \frac{1}{(q-1) G(\bar{N})} \sum_{B} G(\bar{B}) B(2 a) \sum_{u} B \bar{N}(u) \zeta^{u^{2}+2 u x} \\
& =\frac{1}{G(\bar{N})} \sum_{u \neq 0} \bar{N}(u) \zeta^{u^{2}+2 u x} \frac{1}{q-1} \sum_{B} G(\bar{B}) B(2 u a) \\
& =\frac{1}{G(\bar{N})} \sum_{u} \bar{N}(u) \zeta^{u^{2}+2 u x+2 u a}=H_{N}(x+a) .
\end{aligned}
$$


Lemma 4.30. Let $z \neq 0$. Then ( $c f$. Theorem 4.19)

$$
L:=\sum_{t} \zeta^{t^{2}} H_{M}(t) H_{N}(t+z)=G(M \bar{N}) G(\phi) N(2 z) \bar{M}(-z) / G(\bar{N}) .
$$

Proof.

$$
\begin{aligned}
G(\bar{M}) G(\bar{N}) L & =\sum_{s, t, u} \bar{M}(s) \bar{N}(u) \zeta^{t^{2}+s^{2}+2 s t+u^{2}+2 u(t+z)} \\
& =G(\phi) \sum_{s, u \neq 0} \bar{M}(s) \bar{N}(u) \zeta^{2 u z-2 u s} \\
& =G(\phi) \sum_{s, u \neq 0} \bar{M}(s) M \bar{N}(u) \zeta^{2 u z-2 s} \\
& =G(\phi) M(-2) G(\bar{M}) N \bar{M}(2 z) G(M \bar{N}),
\end{aligned}
$$

as desired.

Corresponding to (cf. (4.3) and (4.7))

(4.31) $e^{2 x z-z^{2}} H_{m}(x-z)=\sum_{n=0}^{\infty} \frac{H_{n+m}(x) z^{n}}{n !} \quad[25$, p. 197, (1)],

we have

TheOREM 4.31. Let $z \neq 0$. Then

$$
\begin{aligned}
F(x) & :=\zeta^{z^{2}-2 x z} H_{M}(x-z) \\
& =\frac{\bar{M}(z) \zeta^{-x^{2}} G(\phi)}{G(\bar{M})}+\sum_{N} \frac{G(\bar{N}) N(-z)}{q-1} H_{N M}(x) .
\end{aligned}
$$

Proof. Define $e_{N}$ as in Theorem 4.27. By Lemma 4.30, $e_{N}=$ $N \bar{M}(-z) G(M \bar{N}) /(q-1)$. It is easily proved (cf. Theorem 4.11) that

$$
\sum_{t} \zeta^{-z^{2}-2 t z} H_{M}(t)=q \bar{M}(z) / G(\bar{M}) .
$$

Thus the result follows from Theorem 4.27.

Corollary 4.32. Let $z \neq 0$. Then

$$
\zeta^{z^{2}} H_{M}(z)=\frac{\bar{M}(-z) G(\phi)}{G(\bar{M})}+\sum_{A} \frac{G(\bar{A}) G\left(\bar{A}^{2} M\right) A^{2} \bar{M}(z)}{(q-1) G\left(\bar{A}^{2}\right)} .
$$

Proof. In Theorem 4.31, set $x=0$, replace $z$ by $-z$, and apply Theorem 4.6. 
Lemma 4.33. Let $a \neq 0, \pm 1$. Then ( $c f$. Lemma 4.30, Theorem 4.19)

$$
\begin{aligned}
L & :=\sum_{x} \zeta^{x^{2}} H_{M}(x) H_{N}(a x) \\
& =\left\{\begin{array}{l}
0, \quad \text { if } \bar{M} N \text { is not a square } \\
\frac{M(-2 a) G(\phi)}{G(\bar{N})}\left(A\left(1-a^{2}\right) G(\bar{A})+\phi A\left(1-a^{2}\right) G(\overline{A \phi})\right), \\
\text { if } \bar{M} N=A^{2} .
\end{array}\right.
\end{aligned}
$$

Proof.

$$
\begin{aligned}
L & =\frac{1}{G(\bar{N}) G(\bar{M})} \sum_{s, t} \bar{M}(s) \bar{N}(t) \zeta^{s^{2}+t^{2}} \sum_{x} \zeta^{x^{2}+2 x(s+a t)} \\
& =\frac{G(\phi)}{G(\bar{N}) G(\bar{M})} \sum_{s, t \neq 0} \bar{M}(s) \bar{N}(t) \zeta^{t^{2}\left(1-a^{2}\right)-2 a s t} \\
& =\frac{M(-2 a) G(\phi)}{G(\bar{M})} \sum_{t} M \bar{N}(t) \zeta^{t^{2}\left(1-a^{2}\right)}
\end{aligned}
$$

and the result easily follows.

Corresponding to

$$
a^{n} H_{n}\left(\frac{x}{a}\right)=n ! \sum_{k=0}^{[n / 2]} \frac{H_{n-2 k}(x)\left(1-a^{2}\right)^{k}}{(n-2 k) ! k !}
$$

(of which the special case $a=\sqrt{2}$ is given in [25, p. 253, Ex. 7]), we have (cf. Theorem 4.20)

THEOREM 4.34. Let $a \neq 0, \pm 1$. Then

$$
N(a) H_{N}\left(\frac{x}{a}\right)=\frac{1}{(q-1) G(\bar{N})} \sum_{A} H_{N \bar{A}^{2}}(x) G(\bar{A}) G\left(\bar{N} A^{2}\right) A\left(a^{2}-1\right) .
$$

Proof. By Theorem 4.27 with $F(x)=H_{N}(x / a)$,

$$
H_{N}\left(\frac{x}{a}\right)=\sum_{M} H_{M}(x) \frac{G(\bar{M}) \bar{M}(-2)}{G(\phi)(q-1)} \sum_{t} \zeta^{t^{2}} H_{M}(t) H_{N}\left(\frac{t}{a}\right) .
$$

Applying Lemma 4.33 and replacing $M$ by $N \overrightarrow{A^{2}}$, we obtain the result.

Corresponding to the formula

$$
e^{-t^{2}-2 x t} H_{n}\left(x+t+\frac{a}{t}\right)=\sum_{m=0}^{\infty} L_{m}^{n-m}(2 a) H_{m}(x)\left(\frac{t}{2 a}\right)^{m-n}
$$


a version of which is incorrectly stated in [22, (4.76)], we have (cf. Theorems 4.29 and 4.31)

THEOREM 4.35. For $a t \neq 0$ and $N \neq 1$,

$$
\begin{aligned}
F(t) & :=\zeta^{t^{2}+2 x t} H_{N}(x+t+a \bar{t}) \\
& =\frac{\bar{N}(-t) G(\phi) \zeta^{-x^{2}-2 a}}{G(\bar{N})}+\frac{q}{q-1} \sum_{M} L_{M}^{N \bar{M}}(2 a) H_{M}(x) M \bar{N}\left(\frac{t}{2 a}\right) .
\end{aligned}
$$

Proof. By (2.8),

$$
\begin{aligned}
\left.G(\bar{A}) D_{t}^{A} F(t)\right|_{t=0}=\sum_{u} \bar{A}(u) \zeta^{u^{2}-2 u x} H_{N}(x-u-a \bar{u}) \\
=\frac{1}{G(\bar{N})} \sum_{u, v} \bar{A}(u) \bar{N}(v) \zeta^{u^{2}-2 u x+v^{2}+2 v x-2 v u-2 a v \bar{u}} \\
=\frac{1}{G(\bar{N})} \sum_{u, v} \bar{A}(u) \bar{N}(v+u) \zeta^{v^{2}+2 v x-2 a v \bar{u}-2 a} \\
=\frac{\zeta^{-2 a}}{G(\bar{N})}\left(\sum_{u} \bar{A} \bar{N}(u)-\sum_{u, v \neq 0} \bar{A}(u v) \bar{N}(v+u v) \zeta^{v^{2}+2 v x-2 a \bar{u}}\right) \\
=\frac{\zeta^{-2 a}}{G(\bar{N})}\left(\sum_{u} \overline{A N}(u)+\sum_{u, v} \overline{A N}(v) A N(u) \bar{N}(1+u) \zeta^{v^{2}+2 v x-2 a u}\right) \\
=\frac{\zeta^{-2 a}}{G(\bar{N})}\left(\sum_{u} \overline{A N}(u)+H_{A N}(x) G(\overline{A N}) \sum_{u} A N(u) \bar{N}(1+u) \zeta^{-2 a u}\right) .
\end{aligned}
$$

Now, since $N \neq 1$ and $a \neq 0$,

$$
\begin{array}{rl}
G(\overline{A N}) \sum_{u} A & N(u) \bar{N}(1+u) \zeta^{-2 a u}=\sum_{u, v \neq 0} \overline{A N}(v) \bar{N}(1+u) \zeta^{u(v-2 a)} \\
& =-\sum_{v} \overline{A N}(v)+G(\bar{N}) \sum_{v} \overline{A N}(v) N(v-2 a) \zeta^{2 a-v} \\
& =-\sum_{v} \overline{A N}(v)+\zeta^{2 a} G(\bar{N}) \bar{A}(-2 a) \sum_{v} \overline{A N}(v) N(v+1) \zeta^{2 a v} \\
& =-\sum_{v} \overline{A N}(v)+\zeta^{2 a} G(\bar{N}) \bar{A}(-2 a) q L_{A N}^{\bar{A}}(2 a) .
\end{array}
$$

Thus,

$$
\begin{aligned}
\left.G(\bar{A}) D_{t}^{A} F(t)\right|_{t=0}= & \frac{\zeta^{-2 a}}{G(\bar{N})} \sum_{u} \overline{A N}(u)\left(1-H_{A N}(x)\right) \\
& +H_{A N}(x) \bar{A}(-2 a) q L_{A N}^{\bar{A}}(2 a)
\end{aligned}
$$


Therefore, by Theorem 2.4,

$$
\begin{aligned}
F(t)= & \frac{\zeta^{-2 a}}{(q-1) G(\bar{N})} \sum_{A}\left(1-H_{A N}(x)\right) A(-t) \sum_{u} \overline{A N}(u) \\
& +\frac{q}{q-1} \sum_{A} H_{A N}(x) A\left(\frac{t}{2 a}\right) L_{A N}^{\bar{A}}(2 a) .
\end{aligned}
$$

The result now follows with use of Theorem 4.4.

Let $G_{n}(x)$ denote the Hermite function of the second kind defined in $[2,(2.44)]$. In view of $[\mathbf{2 0},(9.13 .8)$ and $(1.2 .3)]$, it is reasonable to consider $\phi(-1) H_{N}(x)$ as the finite field analogue of $G_{n}(x)$.

Corresponding to (cf. (4.24))

$$
\begin{aligned}
& \int_{-\infty}^{\infty} G_{m+l+2 k+1}(x) H_{m}(x) H_{l}(x) e^{-2 x^{2}} d x \\
& =\frac{(-1)^{k} 2^{k+l+m}(k+m) !(k+l) !}{k !} \quad[2,(2.47)],
\end{aligned}
$$

we have

TheOREM 4.36. Let $A, B, C$, and $A B C$ be nontrivial. Then $L:=\sum_{x} \zeta^{2 x^{2}} H_{A}(x) H_{B}(x) H_{C}(x)$

$$
=\left\{\begin{array}{l}
0, \quad \text { if } A B C \text { is not a square, } \\
\begin{array}{r}
q^{-1} \phi(-1) \bar{N}(2) G(\overline{A N}) G(\bar{B} \bar{N}) G(\overline{C N}) \\
\quad+q^{-1} \phi(-1) \bar{N} \phi(2) G(\overline{A N} \phi) G(\bar{B} \bar{N} \phi) G(\overline{C N} \phi), \quad \text { if } \bar{A} \bar{B} \bar{C}=N^{2} .
\end{array}
\end{array}\right.
$$

Proof. We have

$$
\begin{aligned}
L & =\frac{1}{G(\bar{A}) G(\bar{B}) G(\bar{C})} \sum_{r, s, t, x} \bar{A}(r) \bar{B}(s) \bar{C}(t) \zeta^{r^{2}+s^{2}+t^{2}+2 x^{2}+2 x(r+s+t)} \\
& =\frac{G(\phi) \phi(2)}{G(\bar{A}) G(\bar{B}) G(\bar{C})} \sum_{r, s, t \neq 0} \bar{A}(r) \bar{B}(s) \bar{C}(t) \zeta^{\left(r^{2}+s^{2}+t^{2}-2 r s-2 r t-2 s t\right) / 2} \\
& =\frac{G(\phi) \phi(2)}{G(\bar{A}) G(\bar{B}) G(\bar{C})} \sum_{r, s, t \neq 0} \overline{A B C}(t) \bar{B}(s) \bar{C}(r) \zeta^{t^{2}\left(1+r^{2}+s^{2}-2 r s-2 r-2 s\right) / 2} .
\end{aligned}
$$

Clearly $L=0$ if $A B C$ is not a square, so assume that $\overline{A B C}=N^{2}$. Then, since $N^{2} \neq 1$,

$$
L=\frac{G(\phi) \phi(2)}{G(\bar{A}) G(\bar{B}) G(\bar{C})}(N(2) G(N) Z(N)
$$

$$
+N \phi(2) G(N \phi) Z(N \phi))
$$


where

(4.36b) $Z(N)=\sum_{r, s} \bar{B}(s) \bar{C}(r) \bar{N}\left(1+r^{2}+s^{2}-2 r s-2 r-2 s\right)$.

By [7, (20) and (27)],

(4.36c) $Z(N)=\frac{N \phi(-1) G\left(\bar{N}^{2}\right) G(\bar{B}) G(\bar{C}) J(\bar{B} \bar{N} \phi, \overline{C N} \phi, N \phi)}{q G(A)}$,

where

$$
J\left(M_{1}, M_{2}, M_{3}\right):=\sum_{x+y+z=1} M_{1}(x) M_{2}(y) M_{3}(z) .
$$

It is well-known $[17$, p. 100] that

(4.36d) $J\left(M_{1}, M_{2}, M_{3}\right)=G\left(M_{1}\right) G\left(M_{2}\right) G\left(M_{3}\right) \overline{G\left(M_{1} M_{2} M_{3}\right)} / q$

when $M_{3} \neq 1$. By $(4.36 \mathrm{c})$ and $(4.36 \mathrm{~d})$,

(4.36e)

$Z(N)=\frac{N \phi(-1) G\left(\bar{N}^{2}\right) G(\bar{B}) G(\bar{C}) G(\bar{B} \bar{N} \phi) G(\overline{C N} \phi) G(N \phi) \overline{G(\overline{B C N} \phi)}}{q^{2} G(A)}$,

and $Z(N \phi)$ is found by replacing $N$ by $N \phi$ in (4.36e). Thus, from (4.36a) and (2.15), the result follows.

5. Associated Hermite sums. Let $H_{n}(x ; c)$ denote the associated Hermite polynomial defined in [1, p. 16]. In view of the generating function formulas (4.7) and

$$
\sum_{m=0}^{\infty} \frac{t^{m+c} H_{m}(x ; c)}{(c)_{m+1}}=e^{2 x t-t^{2}} \int_{0}^{t} u^{c-1} e^{u^{2}-2 x u} d u \quad[\mathbf{1},(4.14)]
$$

it is reasonable to define the finite field analogue $H_{M}(x ; C)$ of $H_{m}(x ; c)$, for a nontrivial character $C$, by

$$
H_{M}(x ; C)=H_{M C}(x) \frac{1}{G(C)} \sum_{u} C(u) \zeta^{2 x u-u^{2}} .
$$

Thus,

$$
H_{M}(x ; C)=H_{M C}(x) \overline{H_{C}(x)}=H_{M C}(x) H_{C}(x) \bar{C}(2) \zeta^{x^{2}} q /(G(\phi) G(C)),
$$

where the last equality follows from Corollary 4.10. In view of Theorem 4.19, the character sums $H_{M}(x ; C)$ clearly satisfy an orthogonality relation with weight function $\zeta^{x^{2}} /{\overline{H_{C}(x)}}^{2}$ (cf. [1, (4.7)]).

To obtain the analogue of the polynomial representation of $H_{m}(x ; c)$ in powers of $x$ (see [1, (2.8) and §5]), one must, according to Theorem 2.4, 
compute $D^{N} H_{M}(x ; C)$ at $x=0$. This may be accomplished by applying Theorems 4.28, 4.3, and 4.6. We omit the details.

We close with an example to show how finite field analysis might be used to conjecture explicit formulas for special functions, which then may be proved by complex analytic methods.

Suppose it is desired to find a formula for $H_{m}(x ; c)$ as a line4ar combination of Hermite polynomials. By Theorem 4.27, we have essentially

$$
\begin{aligned}
H_{M}(x ; C) \stackrel{*}{=} & \frac{\bar{C}(2) \phi(-1)}{(q-1) G(C)} \\
& \times \sum_{A} H_{A}(x) G(\bar{A}) \bar{A}(-2) \sum_{t} \zeta^{2 t^{2}} H_{A}(t) H_{C}(t) H_{M C}(t),
\end{aligned}
$$

where the asterisk signifies that a few isolated terms have been ignored. Using Theorem 4.36 to evaluate the inner sum on $t$, we have

$$
\begin{aligned}
H_{M}(x ; C) \stackrel{*}{=} & \frac{\bar{C}(2)}{q(q-1) G(C)} \sum_{N} H_{\bar{B} \bar{C} \bar{N}^{2}}(x) G\left(B C N^{2}\right) \\
& \times B C N^{2}(-2) \bar{N}(2) G(B C N) G(\overline{B N}) G(\overline{C N}),
\end{aligned}
$$

where $B=M C$. Replace $N$ by $N \bar{B}$ to obtain

$$
\begin{aligned}
H_{M}(x ; C) \stackrel{*}{=} & \frac{M(-1)}{q(q-1) G(C)} \\
& \times \sum_{N} H_{M \bar{N}^{2}}(x) G\left(N^{2} \bar{M}\right) N(2) G(N C) G(\bar{N}) G(M \bar{N}) .
\end{aligned}
$$

From this one might conjecture that

$$
H_{m}(x ; c)=\sum_{n=0}^{\infty} \frac{(-2)^{n}(c)_{n}(m-n) !}{n !(m-2 n) !} H_{m-2 n}(x),
$$

which is in agreement with [1, (4.18)].

Acknowledgment. The author is grateful to Richard Askey for several helpful suggestions.

\section{REFERENCES}

[1] R. Askey and J. Wimp, Associated Laguerre and Hermite polynomials, Proc. Royal Soc. Edinburgh, 96A (1984), 15-37.

[2] R. Askey, T. Koornwinder, and M. Rahman, An integral of products of ultraspherical functions and a q-extension, to appear. 
[3] B. Berndt and R. Evans, Sums of Gauss, Eisenstein, Jacobi, Jacobsthal, and Brewer, Illinois J. Math., 23 (1979), 374-437.

[4] G. Birkhoff and S. MacLane, A Survey of Modern Algebra, 3rd edition, Macmillan, N. Y., 1965.

[5] H. Davenport and H. Hasse, Die Nullstellen der Kongruenzzetafunktionen in gewissen zyklischen Fällen, J. reine angew. Math., 172 (1934), 151-182.

[6] A. Erdélyi, Higher Transcendental Functions, vol. 2, McGraw Hill, N. Y., 1953.

[7] R. Evans, Identities for products of Gauss sums over finite fields, Enseignement Math., 27 (1981), 197-209.

[8] _ Character sum analogues of constant term identities for root systems, Israel J. Math., 46 (1983), 189-196.

[9] R. Evans, J. Pulham, and J. Sheehan, On the number of complete subgraphs contained in certain graphs, J. Combinatorial Theory, Series B, 30 (1981), 364-371.

[10] D. Goldfeld and P. Sarnak, Sums of Kloosterman sums, Inventiones Math., 71 (1983), 243-250.

[11] J. Greene, Character sum analogues for hypergeometric and generalized hypergeometric functions over finite fields, Ph.D. thesis, University of Minnesota, 1984.

[11A] J. Greene, Hypergeometric functions over finite fields, preprint.

[12] J. Greene and D. Stanton, A character sum evaluation and Gaussian hypergeometric series, J. Number Theory, (to appear).

[13] _ The triplication formula for Gauss sums, preprint.

[14] A. Helversen-Pasotto, L'identité de Barnes pour les corps finis, C. R. Acad. Sci. Paris, Série A, 286 (1978), 297-300.

[15] _ L'identité de Barnes pour les corps finis, Séminaire Delange-Pisot-Poitou (Théorie des Nombres), 19e année, 22(1977/78).

[16] __ Darstellungen von $G l\left(3, F_{q}\right)$ und Gaußsche summen, Math. Ann., 260 (1982), $1-21$.

[17] K. Ireland and M. Rosen, A Classical Introduction to Modern Number Theory, Springer-Verlag, N. Y., 1982.

[18] C. Jacobi, Über die Kreisteilung und ihre Anwendung auf die Zahlentheorie, J. reine angew. Math., 30 (1846), 166-182; Werke, vol. 6, Chelsea, N. Y., 1969, pp. 254-264.

[19] N. Koblitz, The number of points on certain families of hypersurfaces over finite fields, Compositio Math., 48 (1983), 3-23.

[20] N. Lebedev, Special Functions and Their Applications, Dover, N. Y., 1972.

[21] W. Li and J. Soto-Andrade, Barnes' identities and representations of GL(2), part I: finite field case, J. reine angew Math., 344 (1983), 171-179.

[21A] R. Lidl and H. Niederreiter, Finite Fields, Addison-Wesley, Reading, Mass., 1983.

[22] W. Miller, Lie Theory and Special Functions, Academic Press, N. Y., 1968.

[23] L. Mordell, On some exponential sums related to Kloosterman sums, Acta Arith., 21 (1972), 65-69.

[24] , On Salié's sum, Glasgow Math. J., 14 (1973), 25-26.

[25] E. Rainville, Special Functions, Macmillan, N. Y., 1960.

[26] G. Szegö, Orthogonal polynomials, AMS Colloq. Publ. 23, 4th ed., Providence, R. I., 1975.

[27] N. Vilenkin, Special functions and the theory of group representations, Amer. Math. Soc., Providence, 1968.

Received March 15, 1985. 


\section{PACIFIC JOURNAL OF MATHEMATICS EDITORS}

\author{
V. S. VARAdarajan (Managing Editor) \\ University of California \\ Los Angeles, CA 90024 \\ Hebert Clemens \\ University of Utah \\ Salt Lake City, UT 84112 \\ Charles R. DePrima \\ California Institute of Technology \\ Pasadena, CA 91125
}

R. FINN

Stanford University

Stanford, CA 94305

HeRManN FLASChKa

University of Arizona

Tucson, AZ 85721

RAMESH A. GANGOlli

University of Washington

Seattle, WA 98195

ROBION KIRBY

University of California

Berkeley, CA 94720

\author{
C. C. MOORE \\ University of California \\ Berkeley, CA 94720 \\ H. SAMELSON \\ Stanford University \\ Stanford, CA 94305 \\ HAROLD STARK \\ University of California, San Diego \\ La Jolla, CA 92093
}

\section{ASSOCIATE EDITORS}

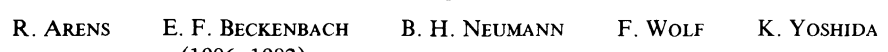

(1906-1982)

\section{SUPPORTING INSTITUTIONS}

\begin{abstract}
UNIVERSITY OF ARIZONA
UNIVERSITY OF BRITISH COLUMBIA

CALIFORNIA INSTITUTE OF TECHNOLOGY

UNIVERSITY OF CALIFORNIA

MONTANA STATE UNIVERSITY

UNIVERSITY OF NEVADA, RENO

NEW MEXICO STATE UNIVERSITY

OREGON STATE UNIVERSITY
\end{abstract}

\author{
UNIVERSITY OF OREGON \\ UNIVERSITY OF SOUTHERN CALIFORNIA \\ STANFORD UNIVERSITY \\ UNIVERSITY OF HAWAII \\ UNIVERSITY OF TOKYO \\ UNIVERSITY OF UTAH \\ WASHINGTON STATE UNIVERSITY \\ UNIVERSITY OF WASHINGTON
}

The Supporting Institutions listed above contribute to the cost of publication of this Journal, but they are not owners or publishers and have no responsibility for its content or policies.

Mathematical papers intended for publication in the Pacific Journal of Mathematics should be in typed form or offset-reproduced (not dittoed), double spaced with large margins. Please do not use built up fractions in the text of the manuscript. However, you may use them in the displayed equations. Underline Greek letters in red, German in green, and script in blue. The first paragraph must be capable of being used separately as a synopsis of the entire paper. In particular it should contain no bibliographic references. Please propose a heading for the odd numbered pages of less than 35 characters. Manuscripts, in triplicate, may be sent to any one of the editors. Please classify according to the scheme of Math. Reviews, Index to Vol. 39. Supply name and address of author to whom proofs should be sent. All other communications should be addressed to the managing editor, or Elaine Barth, University of California, Los Angeles, California 90024.

There are page-charges associated with articles appearing in the Pacific Journal of Mathematics. These charges are expected to be paid by the author's University, Government Agency or Company. If the author or authors do not have access to such Institutional support these charges are waived. Single authors will receive 50 free reprints; joint authors will receive a total of 100 free reprints. Additional copies may be obtained at cost in multiples of 50 .

The Pacific Journal of Mathematics is issued monthly as of January 1966. Regular subscription rate: $\$ 190.00$ a year (5 Vols., 10 issues). Special rate: $\$ 95.00$ a year to individual members of supporting institutions.

Subscriptions, orders for numbers issued in the last three calendar years, and changes of address should be sent to Pacific Journal of Mathematics, P.O. Box 969, Carmel Valley, CA 93924, U.S.A. Old back numbers obtainable from Kraus Periodicals Co., Route 100, Millwood, NY 10546.

The Pacific Journal of Mathematics at P.O. Box 969, Carmel Valley, CA 93924 (ISSN 0030-8730) publishes 5 volumes per year. Application to mail at Second-class postage rates is pending at Carmel Valley, California, and additional mailing offices. Postmaster: Send address changes to Pacific Journal of Mathematics, P.O. Box 969, Carmel Valley, CA 93924.

PUBLISHED BY PACIFIC JOURNAL OF MATHEMATICS, A NON-PROFIT CORPORATION

Copyright $\odot 1986$ by Pacific Journal of Mathematics 


\section{Pacific Journal of Mathematics}

\section{Vol. 122, No. 2 \\ February, 1986}

Gideon Amit and David Chillag, On a question of Feit concerning character values of finite solvable groups ......................257

Constantin Gelu Apostol and Frank Larkin Gilfeather, Isomorphisms modulo the compact operators of nest algebras ................263

Parviz Azimi and James Neil Hagler, Examples of hereditarily $l^{1}$ Banach

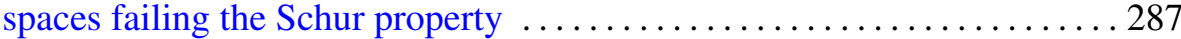

Brian Evan Blank, Boundary behavior of limits of discrete series representations of real rank one semisimple groups . . . . . . . . . . 299

Jeffrey Carroll, Some undecidability results for lattices in recursion theory

Gerald Howard Cliff and Alfred Rheinhold Weiss, Crossed product and

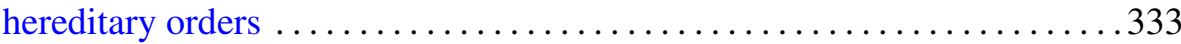

Ralph Cohen, Realizing transfer maps for ramified coverings . . . . . . . . 347

Ronald James Evans, Hermite character sums . .................. 357

C. L. Frenzen and Roderick Sue-Chuen Wong, Asymptotic expansions of the Lebesgue constants for Jacobi series . . . . . . . . . . . . . . . . 391

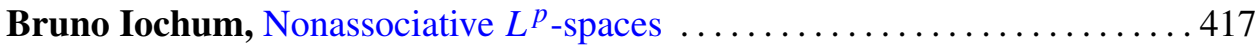

John McDonald, Unimodular approximation in function algebras ....... 435

John Robert Quine, Jr., Ramification and unintegrated value distribution . . 441

Marc Raphael, Commutants of quasisimilar subnormal operators ........ 449

Parameswaran Sankaran and Peter Zvengrowski, On stable

parallelizability of flag manifolds

Helga Schirmer, A relative Nielsen number

Barry Simon, Schrödinger semigroups on the scale of Sobolev spaces . . . . . 475

Viakalathur Shankar Sunder, Stochastic integration in Fock space

Jan de Vries, A note on the $G$-space version of Glicksberg's theorem 